\title{
Analysis on the Security Platform of Status Monitoring and Assessment Technology for Power Grid Equipment
}

\author{
Xiangfu Guo ${ }^{1}$ and Ming Zhong ${ }^{2}$ \\ ${ }^{1}$ State Grid Henan Electric Power Research Institute, 450052 Zhengzhou Henan, China \\ ${ }^{2}$ Zhengzhou Gas Power Generation Co., Ltd. 450052 Zhengzhou Henan, China
}

\begin{abstract}
The high-speed development of society and the constant improvement of living quality requires the construction of power grid facility. To efficiently, conveniently and normally operate the power equipment in the power grid facility to secure the national production development and people's normal production life, the status monitoring and assessment technology for power grid equipment is becoming more and more important. This article mainly introduces the status monitoring technology of power grid equipment and focuses on analysis of the relevant content about the security platform of monitoring and assessment technology.
\end{abstract}

Keywords. power grid equipment; status monitoring; assessment; platform

\section{Definition of status monitoring and assessment technology for power grid equipment}

With the high-speed progress of society, people have an increasingly higher demand on the reliability of the power generation, power transmission, power supply and power consumption in close relation with the production and living of themselves, which also poses a rigorous challenge to the development of power system. The rapid development of social information networking facilitates the development of on-line monitoring, mode identification and processing technology of computer information. The status monitoring of power grid equipment and failure diagnosis technology are also subject to periodical transformation, which is a definite trend for the development of electric appliances ${ }^{[1]}$.

The setting of status monitoring of power grid equipment is a process of monitoring based on the modernized and advanced network information processing technology. This setting is for the convenience of people to timely find out the occurrence time of early-stage failure of power system, and thus the maintenance staff of power system will be able to solve the hidden troubles by detection technology in the early stage of accident about the power equipment or power system, so that the serious accident will be perfectly avoided. The development of power system is always in progress. In the early stage of power system development, the Preventive Experimental Specification for Electric Appliances is always the basis to implement the equipment maintenance for power system.With more dependence of the society on the power equipment, it no longer meets the requirement of the actual society, and the power system is gradually developed to the marketization. The status monitoring and overhaul of electric appliances are drawing more and more attention from people due to the costs reservation and expense reduction. The equipment overhaul system based on the status monitoring and overhaul of power equipment is another breakthrough in the development of power system, and also an important mark for the power maintenance to step to the scientific development. Recently, the application of advanced technology brings a lot of changes in power system. It is believed that in the near future, the status monitoring and assessment technology of power system will usher into a better era. ${ }^{[2-3]}$

\section{Analysis on the security platform of status monitoring and assessment technology for power grid equipment}

\subsection{System development}

The platform project will use the most advanced and maturest route of enterprise-level IT application development technology in the industry, and fully incorporate the demand on the actual business. We will use currently the most optimized solutions for system framework mode, database select, language development, etc.. The system is constructed in $\mathrm{B} / \mathrm{S}$ framework. The Flex+JSP will be used in the foreground interface, and Java in the language development in the background. Based on the framework of Struts 2 and Spring, the ORACLE database will be used. The background uses the map service provided by 
GIS platform in State Grid, and the Flex technology in the foreground will be used to realize the demonstration of figure. This system needs to be integrated with multiple systems, so a large amount of interfaces shall be developed. In case that these interfaces fail to realize the technology in compliance with the standardization, their generality will be greatly impacted, and the afterward created systems will be hard to use those interfaces, which may induce a great number of repeated works and repeated investments. Therefore, the system interface shall be realized by standardized technology.

\subsection{Refining scheme for technology implementa- tion}

\subsubsection{Construction objective of platform}

Complete the data interface related to 7 systems, namely the Production Management System (PMS), Grid Information System (GIS), monitoring system for the status of power transmission and transformation, monitoring and diagnosis platform of chromatograph data center, lightning positioning system, power meteorology system and mountain fire monitoring system. Realize data integration module and integrate the equipment data from each system; The interface will be demonstrated by equipment tree structure, GIS map and comprehensive inquiry list. Process the lightning information, special maps information (lightning area diagram, sewage area diagram, dancing diagram, ice area diagram and wind area diagram), meteorological information and mountain fire information related to the geographical space, which will be demonstrated by the overlapped layers on the geographical background and distribution diagram of power grid. Realize the lightning information used for comprehensive inquiry of routes according to time and distance, and demonstrate them in GIS map dynamically. Regularly make analysis on the result of regular and dynamic assessment about the equipment, monitor the assessment plan and defect status of the equipment, and realize au- tomatic delivery of message precaution by using the existing message platform.

\subsubsection{Content of platform construction}

The content of platform construction shall include the following aspects: data interface development, including the Production Management System (PMS), Grid Information System (GIS), monitoring system for the status of power transmission and transformation, monitoring and diagnosis platform of chromatograph data center, lightning positioning system, power meteorology system and mountain fire monitoring system development. Data integration method for the same equipment among multiple systems. Analysis method for dynamic assessment result of equipment Positioning method of route lightning information in GIS. Conversion of digitalized storage for special maps. Development of assessment status and defect status precaution function of equipment

\subsubsection{Framework of system setup}

The system is divided into 3 parts: basic platform layer, service platform layer and business application layer. In consideration of the variety of data source for the system, we will use the dependency injection features of Spring to register multiple data source in the development, and meanwhile use JDBCTemplate encapsulated by Spring to make the data persistent, as well as record the complex corresponding relation among the data and realize the assembly in the background. After realizing the data assembly in the background, we will return the basic type or objective group of Java to the foreground. Simplify the interactive complexity of data integration module and interface system. As shown in Figure 1.

\subsubsection{Design of system function}

The design of system function shall include the following content: homepage function of system; demonstration of

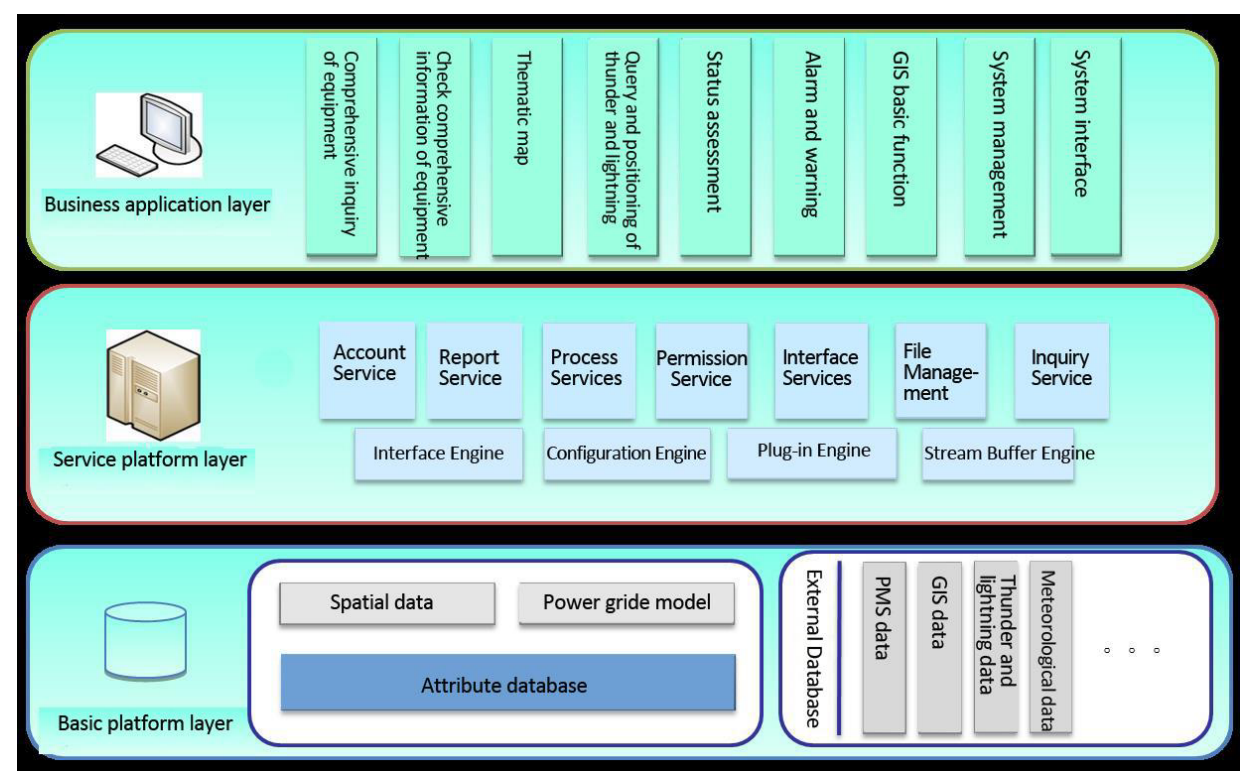

Figure 1. Schematic diagram of framework of system setup. 
comprehensive information for equipment; comprehensive inquiry of equipment: digitalized conversion of special maps; Function of positioning the route lightning information by GIS inquiry; function of status assessment precaution for equipment; data interface, etc.. Due to the limitation of the article, we make analysis on part of the key points.

\section{$A$. Comprehensive inquiry of equipment}

In the inquiry list, the user can enter and select the corresponding conditions in the page and click the inquiry button. Then the system will search all the equipment meeting the conditions entered by user, and demonstrate them in a list. Inquiry conditions provided: equipment type, belonging city, voltage level, defect type, assessment result, etc..

a. Inquiry conditions include:

(1) Equipment type: including transformer substation (auxiliary equipment including transformer, switch, etc.), auxiliary equipment for route machine (poles and towers, insulator, etc.).

(2) Belonging city: data from dictionary items includes: All the 18 cities and provincial overhaul company in Henan Province.

(3) Voltage level: data from dictionary items includes: $220 \mathrm{kV}, 500(330) \mathrm{kV}, 50 \mathrm{kV}, 1000 \mathrm{kV}$

(4) Defect type: data from dictionary items includes: general, serious, emergent.

(5) Assessment result: data from dictionary items includes: excellent, normal.

4 standard classes of State Grid (network, general, standard, hazardous) (Assessment information is once in a year).

(6) Inquiry condition: 5 of the above common conditions are fixedly demonstrated on the page. It is added with advanced inquiry, and the popped out interface will list more inquiry conditions. You may click the inquiry list for refreshment.

b. Inquiry list

The inquiry list will only display general field for various equipment, and the rest of the content will be demonstrated in a popped out page of details by "details" link. The inquiry conditions and list can be hidden. As shown in Figure 2.

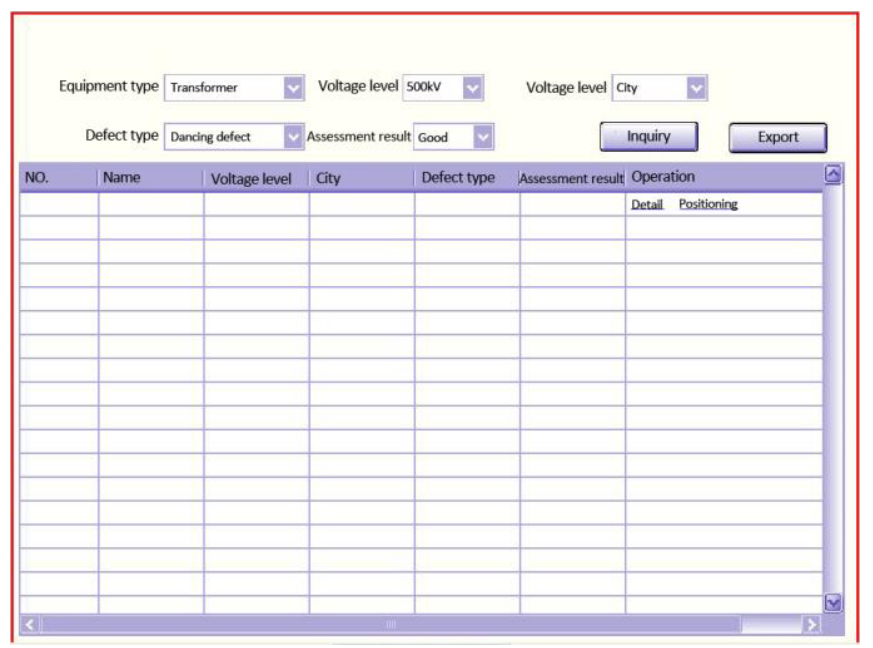

Figure 2. Interface of inquiry list. c. Comprehensive information of equipment

We will generally demonstrate PMS data, on-line monitoring data and other data from multiple systems for a certain equipment. Meanwhile, we may establish a new field attribution for edition and maintenance according to the demand.

$B$. Function of positioning the route lightning information by GIS inquiry

The user may select the route, time period and radius distance of the route in GIS map, and the system will implement the automatic inquiry to display the thunderbolts within the specified range of time and space. Realization method of function: This function is incorporated into the page of inquiry function for equipment. We may select the route in the equipment tree, and right click the menu or a certain button to pop out a dialog of parameter data (including time period, thunderbolts range parameters), then click the inquiry button to refresh the maps and demonstrate the thunderbolts within the specified range of time and space. And we can also select the route on the map by one click on the function button. Then the map will display the thunderbolts within the specified range of time and space.

\section{$C$. Function of status assessment precaution for} equipment

Function of status assessment precaution and warning service for equipment can display the pending information for non-assessed equipment to be expired and the new equipment not assessed for over one month; It will carry out the message warning in case the assessment result of a equipment displays serious status or serious defect status. The mobile phone number is to be provided by user, and the binding rules shall be confirmed according to the belonging conditions of units and groups. The regular assessment date of equipment may be maintained by the system and granted with a uniform value ${ }^{[4]}$.

\section{$D$. Data interface}

All the interfaces will be realized by using Web Service technology and XML technology. Lightning positioning data, power meteorology system, oil chromatograph and on-line monitoring data of fine meteorology: Due to the limited amount of data, we will carry out the synchronization once a day, and use the scheduling system of Spring framework to obtain the new data from the interface in the early-morning every day, and to be incrementally synchronized to local database.

\section{Summary}

In general, the power system is a key factor for national interests and people's livelihood, and the status monitoring of power grid equipment is the most powerful security system for power system, so it shall be focused on. Paying attention to the development trend of status monitoring of power grid equipment and strengthening the study and inspiration therefrom is the key for the technical development of status monitoring of power grid equipment, which may have a promising development prosperity in the future.

\section{References}


1. Xu Xingke. Application of status monitoring and failure diagnosis technology in the equipment management in power plant [J]. Power Equipment, 2005, (03):120-121.

2. Li Qiang. Development trend of status monitoring and failure diagnosis technology for power equipment[J]. Power Informationization, 2009, (02):11-13.
3. Jin Yi, Liu Wei, Zha Xianguang \& Fei Yijun. Application of status monitoring technology for smart transformer substation [J]. Jiangsu Electromechanical Engineering, 2012, (02):20-23.

4. Deng Youzhi. Brief discussion on status monitoring of power equipment[J]. Private-owned Science, 2009 (02):9 